\title{
DYNAMIC RESPONSE OF REAL OFFSHORE WIND TURBINES ON MONOPILES IN STRATIFIED SEABED
}

\author{
Guillermo M. Álamo ${ }^{1}$, Juan J. Aznárez ${ }^{1}$, Luis A. Padrón ${ }^{1}$, Alejandro E. \\ Martínez-Castro $^{2}$, Rafael Gallego ${ }^{2}$ and Orlando Maeso ${ }^{1}$ \\ ${ }^{1}$ Instituto Universitario de Sistemas Inteligentes y Aplicaciones Numéricas en Ingeniería (SIANI), \\ Universidad de Las Palmas de Gran Canaria \\ Edificio Central del Parque Científico y Tecnológico, Campus Universitario de Tafira, 35017 Las \\ Palmas de Gran Canaria, Spain \\ e-mail: \{guillermo.alamo, juanjose.aznarez, luis.padron, orlando.maeso\}@ulpgc.es \\ ${ }^{2}$ Departamento de Mecánica de Estructuras e Ingeniería Hidráulica, ETS de Ingenieros de Caminos, \\ Canales y Puertos, Universidad de Granada \\ Avenida Fuentenueva s/n, 18002 Granada, Spain \\ e-mail: \{amcastro, gallego $@ @$ ugr.es
}

Keywords: offshore wind turbines, soil-structure interaction, monopile impedance functions, modal analysis

\begin{abstract}
This paper studies the influence of the superstructure and foundation parameters of offshore wind turbine (OWT) systems, as well as of the soil profile, on the magnitude of the associated soil-structure interaction (SSI) phenomena. The analyses are carried out by assuming characteristic properties of real OWTs and of soil profiles based on North Sea boreholes. To do so, a simplified substructuring model is proposed for the computation of the fundamental frequency and equivalent damping of OWTs founded on monopiles including the SSI effects. The whole superstructure is reduced to a three-degrees-of-freedom system through its modal mass and height, while the foundation stiffness is represented by impedance functions. The pile impedance functions are computed by a time-harmonic integral model that makes use of Green's Functions for the layered halfspace to represent the soil behaviour, while the pile is represented by finite elements as a Timoshenko beam and treated as a load-line within the soil.

The obtained results confirm the necessity of considering the SSI effects for an accurate estimation of both the fundamental frequency and equivalent damping of the soil-structure system. Regarding the pile dimensions, the pile diameter plays a significant role on the magnitude of the SSI effects, while the pile length has almost no influence. On the other hand, the results highlight the importance of a good knowledge of the soil profile, as high differences are produced between the homogeneous and variable-with-depth profiles, even when both present the same mean shear velocity. The superficial soil layers are found to be the ones of crucial importance when evaluating the SSI effects on the dynamic properties of OWT systems.
\end{abstract}




\section{INTRODUCTION}

Wind energy has become in the last decades a viable and sustainable solution to the continuously increasing energy demand. Offshore wind turbines (OWT) present some advantages over their onshore counterparts: better wind conditions, terrains with less occupancy, and lower noise and visual impact on the population. This, together with the advances in the generators technology, has produced a significant increment of offshore wind farms in the recent times. Among the different foundations systems that are used for OWT constructions, the monopile is preferred over the jacket or gravity-based foundations due to its simplicity and cost-efficiency.

The accurate estimation of the fundamental frequency of the OWT system is a keystone of the design stage of these constructions. The fundamental frequency has to be kept away from the excitation frequency content in order to prevent resonance effects. There are three principal frequencies to avoid: the wind and wave frequencies $(<0.1 \mathrm{~Hz})$, the rotor frequency $(1 \mathrm{P})$ and the blade-passing frequency ( $2 \mathrm{P}$ or $3 \mathrm{P}$ ). Recommendation codes advise to place the system fundamental frequency outside the $\pm 10 \%$ range of the aforementioned frequencies [1]. In order to correctly estimate the tower-foundation system fundamental frequency, the soil stiffness has to be taken into account as the soil-structure interaction (SSI) effects can significantly reduce the system fundamental frequency value. The dynamic characterization, i.e. computation of the modified fundamental frequency and damping, of OWT structures including the SSI effects has been the object of study for numerous recent works [2-21].

In his early work, Zaaijer [2] compared different methodologies used to estimate the structural fundamental frequency taking into account the soil effects. A Finite Element Method (FEM) model including the American Petroleum Institute (API) p-y, t-z, Q-z curves was taken as reference method and different foundation systems, such as monopiles, gravity footings and jackets, were assumed. The best results were obtained by using impedance matrices, as inertia effects in the foundation and non-linear soil-structure interaction were negligible. Thus, stiffness matrix models are applicable for pile foundations under loading conditions relevant for the fatigue analysis. The obtained numerical results were compared with measures from two wind farms, resulting in acceptable predictions of the fundamental frequencies. However, Zaaijer found that, as known from offshore practice, the models tend to underpredict the foundation stiffness and, therefore, the system fundamental frequency. In the same work, Zaaijer also studied the sensitivity of the system fundamental frequency to variations in different parameters of soil, structure, foundation and environment, obtaining that soil parameters dominate the uncertainty of the natural frequency. The effect of the uncertainties in the soil profile was also studied by Carswell et al. [8] through probabilistic methods focusing on the Serviceability Limit State (SLS). They concluded that the system reliability shows the same sensibility to load as to soil uncertainty.

Adhikari and Bhattacharya [3] enhanced the Bernoulli model developed by Tempel and Molenaar [22] in order to include the effects of a flexible foundation and the tower axial load. The pile foundation was represented by two frequency-independent springs simulating the lateral and rotational stiffness. They illustrated the analytical results with numerical examples and applied their model to real turbines. Later Bhattacharya and Adhikari [9] evaluated the lateral and rotational foundation stiffness by direct measurement and compared their results with those obtained from a small-scale prototype and from a FEM model. They observed that analytical and FEM results overestimated, in general, the system natural frequency. More recently, Arany et al. [4] further developed their model by including the cross-coupling term in the foundation stiffness and using the Timoshenko theory to model the tower. They concluded 
that the cross-coupling spring term has a significant effect on the natural frequency, while the Timoshenko beam model does not significantly improve the results, being the slender beam assumption accurate enough. The effect of including the cross-coupling term was also studied by Zania [5]. She presented an analytical iterative method to obtain the equivalent modified period and damping due to SSI effects based on the pile impedance functions from Novak and Nogami [23]. She concluded that disregarding the off-diagonal terms and the frequency dependency of the impedance matrix is inappropriate, since it results in a non-conservative overestimation of the fundamental frequency and underestimation of damping. This effect was more evident as the height of the system increases. This conclusion might explain why Bhattacharya and Adhikari [9] find that natural frequencies tend to be overestimated, disagreeing with what was previously exposed by Zaaijer [2].

One of the drawbacks of using the impedance functions is that they are intrinsically defined in the frequency-domain. Implementation into time-domain models allowing non-linear analysis can be done by adopting methodologies such as lumped-parameter models (LPM) [24], as done by Damgaard et al. [10, 11]. They developed different LPM to represent the impedance functions of gravity [10] and monopile [11] foundations and implemented such models in the aeroelastic code HAWC2. By studying the reference NREL 5MW OWT [25], they concluded that the side-side response is more affected by the SSI than the fore-aft vibration for gravity foundations; and that the SSI effects are critical in the design of OWT on monopiles as they have a great impact on the fatigue damage equivalent moment at seabed. Their LPM was used in a later work [12] to study the effect of changes in the soil properties on the system fundamental frequency, damping and fatigue loads in parked conditions. The changes of soil stiffness, soil damping and the presence of sediment transportation at seabed were shown to be critical.

Bisoi and Haldar [6] made use of the p-y curves to represent the soil-pile interaction in a FEM model that included wind and wave loads. They compared three soil profiles (homogeneous, linear and parabolic) obtaining that the natural frequency marginally changes between them. The three profiles presented the same properties at a depth equal to the pile diameter. They also found that the effect of soil non-linearity increases for higher wind speeds. Damgaard et al. [13] obtained the OWT fundamental frequency and damping by using both experimental data from rotor-stop tests and a Winkler approach based on the p-y curves. The Winkler approach together with a hysteresis loop method reasonably estimated the modal soil material damping.

Bhattacharya et al. [14] carried out small scale tests of OWT founded on monopiles and tripods. They illustrated that the natural frequency of the overall system shifts with the number of cycles of loading due to the softening or stiffening of the foundation. Lombardi et al. [7] further studied this relation for monopiles on clay soils, obtaining that the fundamental frequency decreases while the damping increases with the number of cycles of repeated loading. The drop in the natural frequency is higher when the forcing frequency is close to the system natural frequency.

Damgaard et al. [15] studied the influence of the water pore pressure on the estimation of the tower fundamental frequency by combining a Kelvin and a bidimensional FEM models. They compared the numerical results with experimental free-vibration tests, obtaining a better agreement when the permeability of the soil was considered. Yu et al. [16] also investigated the effect of the presence of water in the soil on the dynamic behaviour of OWT founded on monopiles and gravity foundations by executing earthquake centrifuge tests. They demonstrated that the SSI plays a significant role in the seismic behaviour of OWT, affecting the structural settlement, foundation response and fundamental frequency of the system. They remarked the difficulty of the observation and analysis, specially if soil liquefaction is produced. 
Bisoi and Haldar [17] addressed the optimization of the structural mass for 2 and $5 \mathrm{MW}$ OWT founded on monopiles in clay. The SLS, fatigue life and resonance avoidance criteria were checked and p-y curves were used to model the soil-pile interaction. They found that the rotor and nacelle mass and the tower height play a crucial role on design, while the embedded depth of the monopile beyond the critical length has a marginal impact. Myers et al. [18] analysed when the strength (resistance in operational and extreme conditions) or stiffness (resonance avoidance) criteria govern the design of monopiles for OWT, and presented optimum pile sections that satisfied these demands. If a fixed base was assumed, the strength criterion controlled the design; but when the soil flexibility was included, the stiffness criterion became important in two of the three studied sites, corresponding to deeper water depths.

Despite most of the studies focus on the tower lateral vibrations, there are several works related to other vibration modes. Kjørlaug and Kaynia [19] studied the vertical seismic response of the NREL 5MW OWT, showing that the tower could amplify up to two times the vertical accelerations at the seabed. On the other hand, Tibaldi et al. [20] showed that, in addition to the structural modes, the blade and additional aeroelastic modes can play a significant role in the structural response during operational conditions.

An interesting associated phenomenon is highlighted, for instance, by $\mathrm{Hu}$ et al. [21], who showed the tendency of the system to easily get stuck in resonance, exposing the necessity of a good estimation of the system fundamental frequency in generators with variable rotor speed.

\section{PROBLEM DEFINITION}

OWT structures founded on monopiles consist of the assembly of three parts: the tower, a transition piece and the pile. The tower is usually a conical hollow beam that supports the rotor and generator nacelle. The monopile is a cylindrical hollow beam that is driven into the seabed, so part of it lays under the soil while another part stays submerged and also can reach the sea level. Both the tower and monopile are connected through a transition piece, being the access point to the structure, usually employed for maintenance and repair activities.

The whole system can be divided into two different parts: the foundation and the (super)structure. The foundation corresponds to the pile length embedded in the seabed and is the part of the system that interact with the soil. On the other hand, the structure corresponds to the part of the system above soil. In some cases, only the structure is analysed during the design stage of the OWT system by considering an infinitely rigid base. If this assumption is made, the dynamic behaviour of the superstructure can be represented by its fixed-base fundamental frequency $f_{n}$ and modal damping ratio $\xi$. However, as the soil does not behave as an infinitely rigid body, the SSI effects modify the foundation-structure dynamic properties: reducing the fundamental frequency and changing (increasing or decreasing) the damping ratio. This work aims to quantify these changes by computing the system fundamental frequency $\tilde{f}_{n}$ and equivalent damping ratio $\tilde{\xi}$ including the SSI effects.

\subsection{Structure definition based on real OWT data}

As mentioned before, the superstructure consists of three components: tower, transition piece and monopile (above soil). A set of real OWT structures that have been studied in the literature is taken as starting point in order to stablish the dimensions and properties of the systems under analysis. The first group (OWTs 1-12) corresponds to different constructions from wind farms built in the United Kingdom [26]; while OWTs 13-16 are four OWT systems that have been recursively studied by some authors [2-77]. As no detailed information was accessible about the 
dimensions of the transition piece, in this study only the tower and pile lengths are considered.

The tower is defined by the tower heigh $H_{t}$, tower base $D_{\text {bot }}$ and tip $D_{\text {top }}$ external diameters, and tower thickness ratio $\delta_{t}$, which represents the ratio between the inner and outer diameters of the tower cross-section. The thickness ratio is assumed to present the same value along the tower, so thicker walls are located at the tower base. The mass of the rotor and nacelle components is represented by a punctual mass $M_{R N A}$. Table 1 shows the values of the tower variables for the set of OWT systems analysed.

\begin{tabular}{clccccc}
\hline & OWT & $M_{R N A}\left(10^{3} \mathrm{~kg}\right)$ & $H_{t}(\mathrm{~m})$ & $D_{\text {top }}(\mathrm{m})$ & $D_{\text {bot }}(\mathrm{m})$ & $\delta_{t}(\%)$ \\
\hline $1-2$ & Vestas 2MW-V66 & 80 & $60-78$ & 2.3 & 4.2 & 98.0 \\
$3-4$ & Vestas 3MW-V90 & 111 & $8-105$ & 2.3 & 4.2 & 98.0 \\
$5-6$ & Vestas 2MW-V80 & 94 & $60-100$ & 2.3 & 4.2 & 98.0 \\
$7-8$ & Siemens SWT-3.6-107 & 220 & $80-96$ & 2.3 & 4.2 & 98.0 \\
$9-10$ & Vestas 2MW-V80 & 94 & $60-100$ & 2.3 & 4.2 & 98.0 \\
$11-12$ & Siemens SWT-3.6-107 & 220 & $80-96$ & 2.3 & 4.2 & 98.0 \\
13 & Lely A2 NM41-2bladed & 32 & 41.5 & 1.9 & 3.2 & 99.0 \\
14 & North Hoyle 2MW-V80 & 100 & 70 & 2.3 & 4.0 & 97.6 \\
15 & Irene Vorrink 600kW & 36 & 51 & 1.7 & 3.5 & 99.0 \\
16 & Walney S1 3.6MW & 234 & 83.5 & 3.0 & 5.0 & 97.9 \\
\hline
\end{tabular}

Table 1: Tower dimensions for the studied OWT systems.

The monopile is defined by the above-soil pile height $H_{p}$, the pile embedded length $L_{p}$, the pile external diameter $D_{p}$, and the pile thickness ratio $\delta_{p}$, which represents the ratio between the inner and outer diameters of the pile cross-section. Table 2 shows the values of the pile variables for the set of OWT systems analysed. Due to lack of specifications, the pile height is assumed to be equal to the water level. Both the tower and monopile are assumed to be made of steel (Young's modulus $E=210 \mathrm{GPa}$, density $\rho=7850 \mathrm{~kg} / \mathrm{m}^{3}$ and Poisson's ratio $\nu_{p}=0.25$ ).

\begin{tabular}{clcccc}
\hline & \multicolumn{1}{c}{ OWT } & $H_{p}(\mathrm{~m})$ & $L_{p}(\mathrm{~m})$ & $D_{p}(\mathrm{~m})$ & $\delta_{p}(\%)$ \\
\hline $1-2$ & Vestas 2MW-V66 & 11 & 15 & 3.5 & 97.4 \\
$3-4$ & Vestas 3MW-V90 & 10 & 28 & 4.3 & 97.9 \\
$5-6$ & Vestas 2MW-V80 & 20 & 31 & 4.2 & 97.6 \\
$7-8$ & Siemens SWT-3.6-107 & 19 & 11 & 4.7 & 97.7 \\
$9-10$ & Vestas 2MW-V80 & 21 & 33 & 4.0 & 98.2 \\
$11-12$ & Siemens SWT-3.6-107 & 25 & 30 & 4.7 & 97.7 \\
13 & Lely A2 NM41-2bladed & 4.6 & 21 & 3.7 & 98.1 \\
14 & North Hoyle 2MW-V80 & 11 & 33 & 4.0 & 97.5 \\
15 & Irene Vorrink 600kW & 3.8 & 19 & 3.5 & 98.4 \\
16 & Walney S1 3.6MW & 20 & 31 & 4.2 & 97.6 \\
\hline
\end{tabular}

Table 2: Monopile dimensions for the studied OWT systems.

\subsection{Foundation definition based on real OWT data}

The foundation consists of the portion of the monopile embedded in the seabed. Thus, the same properties that are shown in Table 2 define the dimensions of the foundation of each OWT system. 
As can be seen from the collected data, there is no apparent relation between the dimensions of the structure and foundation or between the pile diameter and embedment length. In order to contemplate different possibilities and to study the effect of the foundation dimensions on the SSI effects, a set of three pile diameter values $\left(D_{p}=3,4\right.$ and $\left.5 \mathrm{~m}\right)$ combined with a set of three pile embedment lengths $\left(L_{p}=15,25\right.$ and $\left.35 \mathrm{~m}\right)$ will be considered in the parametric analysis carried out in section 5. The pile thickness ratio is obtained as a function of the pile diameter following the API recommendations [27]:

$$
\delta_{p} \approx 98-\frac{1.274}{D_{p}}
$$

Finally, as the Timoshenko beam theory is used to model the pile (see section 3.2), the shear coefficient $\alpha_{p}=0.5$ for hollow circular cross-sections is assumed.

\subsection{Soil profile definition based on real boreholes}

Attempting to study the SSI effects for soil profiles close to real seabed, two typical boreholes (Nelson Field and Hutton TLP) of the North Sea [28] are selected as reference profiles in this work. As these soils consist of different layers of clay and sand, two additional soil profiles formed only by clay or sand are also included in the analyses. The profiles are presented in terms of the shear wave propagation velocity $c_{s}$, which is obtained as a function of the material and depth $z$ through the expression provided by Ohta and Goto [29]:

$$
c_{s}(z)=78.98 z^{0.312}\left(\begin{array}{ll}
1.000 & \text { clay } \\
1.260 & \text { fine sand } \\
1.286 & \text { medium sand }
\end{array}\right) \quad(\mathrm{m} / \mathrm{s})
$$

Fig. 1 shows the evolution with depth of the aforementioned soil profiles together with their mean value $c_{s, 30}$ [30], as this value is a common choice to characterize the soil. Additionally, a homogeneous profile with $c_{s}=180 \mathrm{~m} / \mathrm{s}$ (close to the Nelson Field mean shear velocity) is also included in the study in order to contrast whether the homogeneous assumption is a valid option.

For depths greater than $80 \mathrm{~m}$, the shear velocity is kept constant with depth (halfspace domain). For all profiles, depth-independent soil density $\rho_{s}=1800 \mathrm{~kg} / \mathrm{m}^{3}$, soil Poisson's ratio $\nu_{s}=0.35$ and soil hysteretic damping ratio $\xi_{s}=5 \%$ are assumed. Nevertheless, in section 5.1 the influence of the soil Poisson's ratio is studied by varying its value between $0.35-0.49$, as higher ratios are commonly used to represent saturated soils.
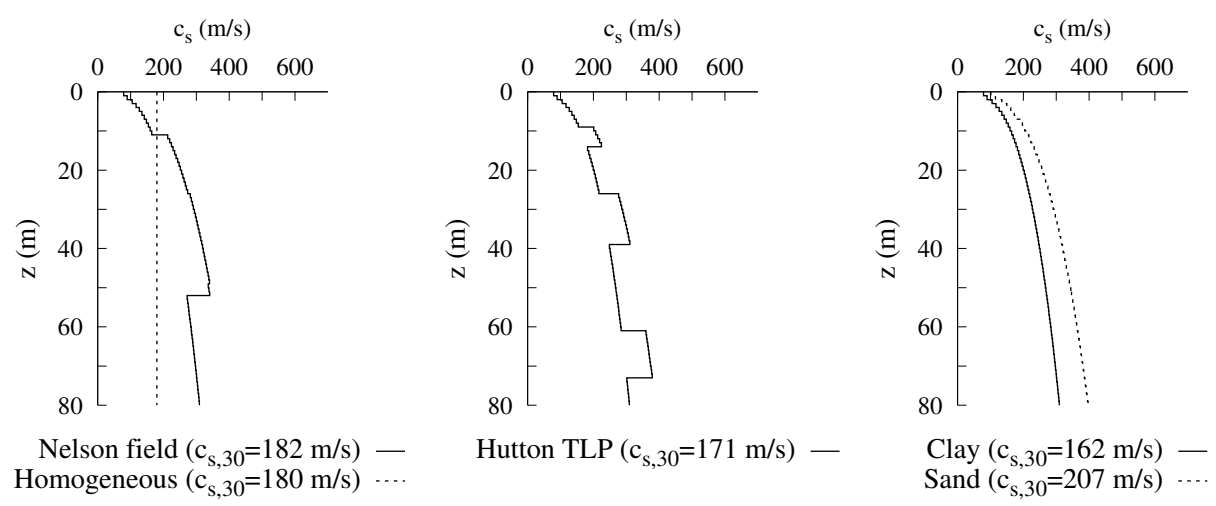

Figure 1: Soils profiles used in the study. Evolution with depth of the shear wave velocity and $c_{s, 30}$ mean value. 


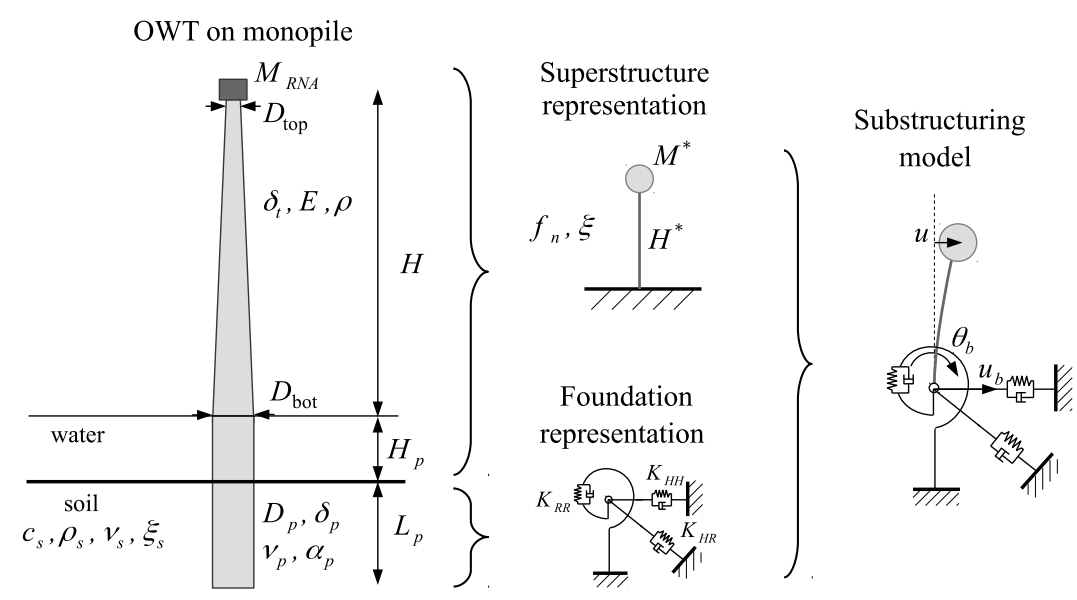

Figure 2: Three-step methodology illustration.

\section{METHODOLOGY}

Fig. 2 depicts the proposed three-step methodology for the dynamic characterization of the foundation-structure system: on one hand, the superstructure is represented by a singledegree-of-freedom whose mass, height and stiffness are determined by the modal properties of the OWT structure; on the other hand, the monopile foundation stiffness is represented by a set of impedance functions. The scope of this study is to analyse the lateral fundamental mode of vibration, so both steps can be reduced to two-dimensional problems. Finally, both the superstructure and foundation representations are coupled together into the final substructuring model.

\subsection{FEM formulation for the modal characterization of the structure}

In order to obtain the mass and height that will characterise the superstructure system a modal analysis is made based on a FEM representation of it. The choice of FEM instead of using analytical expressions is made due to the combination of the tapered (tower) and cylindrical (pile) sections of the OWT structure. Furthermore, with the FEM one can easily assume different properties for each element in order to include additional mass or stiffness due to constructive elements. As the effects of the foundations are not included at this step, the structure is assumed to be placed on an infinitely rigid base.

The structure is modelled as Bernoulli beams by using two-node Hermitian elements with four degrees-of-freedom corresponding to the lateral displacements and rotations. An enough number of constant-section elements are used for both the conical and cylindrical lengths, based on a convergence study. The rotor and nacelle punctual mass $M_{R N A}$ is included at the tower tip node. For the submerged pile elements, the hydrodynamic water added mass and the mass of the water inside the pile are also included through a modified density. This mass inclusion does not affect the results of the fundamental mode of vibration [5], even though the system total mass is significantly increased.

Considering time-harmonic displacements and forces, the system equation of motion results in:

$$
(\mathbf{K}-\omega \mathbf{M}) \mathbf{u}=\mathbf{F}_{\text {ext }}
$$

where $\mathbf{K}$ and $\mathbf{M}$ are the global stiffness and mass matrices obtained by the assembly of the elemental ones; $\mathbf{u}$ is the vector of nodal displacements and rotations and $\mathbf{F}_{\text {ext }}$ is the vector of 
external nodal loads. The structure modal analysis is made by solving the eigenvalues problem $\left|\mathbf{K}-\omega^{2} \mathbf{M}\right|=0$, so the fixed-base fundamental frequency $\omega_{n}$ and its modal shape $\phi_{n}$ are obtained as the smallest eigenvalue and its eigenvector respectively. Then, the base shear effective modal mass $M^{*}$ and height $H^{*}$ of the structure to a base acceleration excitation [31] are computed as:

$$
M^{*}=\frac{\left(\phi_{n}^{T} \mathbf{M} \iota\right)^{2}}{\phi_{n}^{T} \mathbf{M} \phi_{n}} ; \quad H^{*}=\frac{\mathbf{h}^{T} \mathbf{M} \phi_{n}}{\boldsymbol{\phi}_{n}^{T} \mathbf{M} \iota}
$$

being $\iota$ the influence vector and $h$ the height vector (having the node height in the displacement terms and unitary value in the rotation ones). The base shear effective modal mass represents the mass of a single-degree-of-freedom that is equivalent to the studied system in producing the same base shear force at the fundamental frequency. The base shear effective modal height corresponds to the height of the aforementioned modal mass for which the modal mass vibrating at the fundamental frequency produces the same base overturning moment as the studied system. This modal parameters are chosen to represent the whole structure as the base shear force and moment are the excitation forces that affect the foundation.

\subsection{Foundation impedance functions}

The foundation stiffness is modelled through a set of four impedance functions: the horizontal $K_{H H}$, rocking $K_{R R}$ and coupled horizontal-rocking $K_{H R}, K_{R H}$ terms. Each term $K_{i j}$ relates the force or moment in direction $i$ with the displacement or rotation in direction $j$. The impedance functions are frequency-dependent and its real and imaginary components correspond to the stiffness and damping terms, respectively.

For the computation of the monopile impedance functions, a previously developed threedimensional time-harmonic model [32] is used. The model represents the soil behaviour through an integral formulation based on the dynamic reciprocal theorem and making use of Green's Functions for the multilayered halfspace [33], while the pile is modelled as a Timoshenko beam though FEM. Both formulations are coupled assuming that the pile is a load line acting within the soil and imposing compatibility (welded boundary condition in the pile-soil interface) and equilibrium (on the pile-soil interaction tractions) conditions, resulting in a system of linear equations where the pile displacements, pile head external forces and soil-pile interaction tractions are the system unknowns. In order to compute each impedance term, the corresponding pile head displacements are prescribed and the system of equations is solved for the pile head forces.

\subsection{Substructuring simplified model for the dynamic characterization of the system}

At this point, the structure and foundation have been reduced into a single-degree-of-freedom mass and a set of impedance functions respectively. Both are coupled together resulting in a three degrees-of-freedom model where the unknowns are the mass deflection $u$ relative to the base and the base lateral displacement $u_{b}$ and rotation $\theta_{b}$ (see Fig. 2). In order to find the dynamic characteristics of the system, a ground lateral acceleration is assumed as the external excitation yielding the following equations of motion:

$$
\left\{\begin{array}{l}
K^{*} u-M^{*} \omega^{2}\left(u+u_{b}+H^{*} \theta_{b}\right)=-M^{*} \dot{u}_{g} \\
K_{H H} u_{b}+K_{H R} \theta_{b}-M^{*} \omega^{2}\left(u+u_{b}+H^{*} \theta_{b}\right)=-M^{*} \ddot{u}_{g} \\
K_{R H} u_{b}+K_{R R} \theta_{b}-M^{*} \omega^{2} H^{*}\left(u+u_{b}+H^{*} \theta_{b}\right)=-M^{*} \ddot{u}_{g} H^{*}
\end{array}\right.
$$


The term $K^{*}=\omega_{n}^{2} M^{*}(1+2 \mathrm{i} \xi)$ represents the structural lateral stiffness corresponding to the fundamental mode. The structural damping is also represented by its complex component as the modal damping factor $\xi$ is included. The lateral ground acceleration $\ddot{u}_{g}$ is used as the system excitation as the modal mass and height are obtained under the same assumption.

An equivalent single-degree-of-freedom oscillator reproducing the system response is searched for computing the flexible-base fundamental frequency $\tilde{\omega}_{n}$ and damping $\tilde{\xi}$ of the system [34, 35]. An hysteretically damped oscillator is assumed and the equivalence is established in terms of the maximum value of the base shear force per effective seismic force [31] transfer function :

$$
Q(\omega)=\left|\frac{\omega_{n}^{2} u}{\ddot{u}_{g}}\right| \approx\left|\frac{1}{\left(1-\omega^{2} / \tilde{\omega}_{n}^{2}\right)+2 \mathrm{i} \xi}\right|
$$

By iteratively solving eq. 5, the flexible-base fundamental frequency is obtained as the frequency at which the maximum value of the transfer function $Q_{m}$ is produced, while the equivalent damping factor is calculated as $\tilde{\xi}=1 /\left(2 Q_{m}\right)$.

The proposed simplified model is validated against an enhanced FEM model including the foundation stiffness. For that purpose, eq. 3 is modified by adding into the stiffness matrix the foundation impedance functions in the terms corresponding to the ground node, and by assuming an horizontal base acceleration as excitation $\mathbf{F}_{\text {ext }}=-M \iota \ddot{u}_{g}$. Then, the flexible-base fundamental frequency is obtained as the frequency where the maximum value of $Q(\omega)$ takes place. Differences below 1\% are produced for the studied soils between the proposed methodology and the enhanced FEM formulation, revealing the ability of the three-step procedure to correctly capture the foundation-structure system fundamental frequency.

\section{MODAL CHARACTERIZATION OF THE STUDIED OWT STRUCTURES}

Table 3 shows the modal parameters obtained for the set of studied OWT structures once the procedure presented in section 3.1 is applied. Most of the constructions present fixed-base fundamental frequencies between $0.2-0.5 \mathrm{~Hz}$, agreeing with the typical range for medium-sized OWT constructions. Additionally, OWTs 14 and 16 present higher fundamental frequencies due to their limited size (see Table 1) that makes them more rigid structures. The modal height and mass are strongly related to the physical dimensions of the structure, being the first about $85-90 \%$ of the system total height and the later around $30 \%$ of the system total mass.

\begin{tabular}{cccc}
\hline OWT & $f_{n}(\mathrm{~Hz})$ & $H^{*}(\mathrm{~m})$ & $M^{*}\left(10^{3} \mathrm{~kg}\right)$ \\
\hline 1 & 0.53 & 60.8 & 170 \\
2 & 0.37 & 75.4 & 188 \\
3 & 0.35 & 80.1 & 206 \\
4 & 0.23 & 100 & 232 \\
5 & 0.49 & 66.3 & 216 \\
6 & 0.24 & 100 & 239 \\
7 & 0.25 & 91.0 & 329 \\
8 & 0.19 & 105 & 341 \\
\hline
\end{tabular}

\begin{tabular}{cccc}
\hline OWT & $f_{n}(\mathrm{~Hz})$ & $H^{*}(\mathrm{~m})$ & $M^{*}\left(10^{3} \mathrm{~kg}\right)$ \\
\hline 9 & 0.42 & 65.2 & 236 \\
10 & 0.22 & 98.7 & 258 \\
11 & 0.24 & 94.3 & 355 \\
12 & 0.19 & 109 & 363 \\
13 & 0.83 & 43.2 & 46 \\
14 & 0.42 & 70.8 & 203 \\
15 & 0.61 & 51.3 & 51 \\
16 & 0.26 & 89.1 & 469 \\
\hline
\end{tabular}

Table 3: Modal parameters for the studied OWT systems.

A strong relation between the modal parameters and the fixed-base fundamental frequency is found when analysing the obtained results. Thus, a second order polynomial fitting can be used to express the modal mass and height as functions of the fixed-base fundamental frequency 

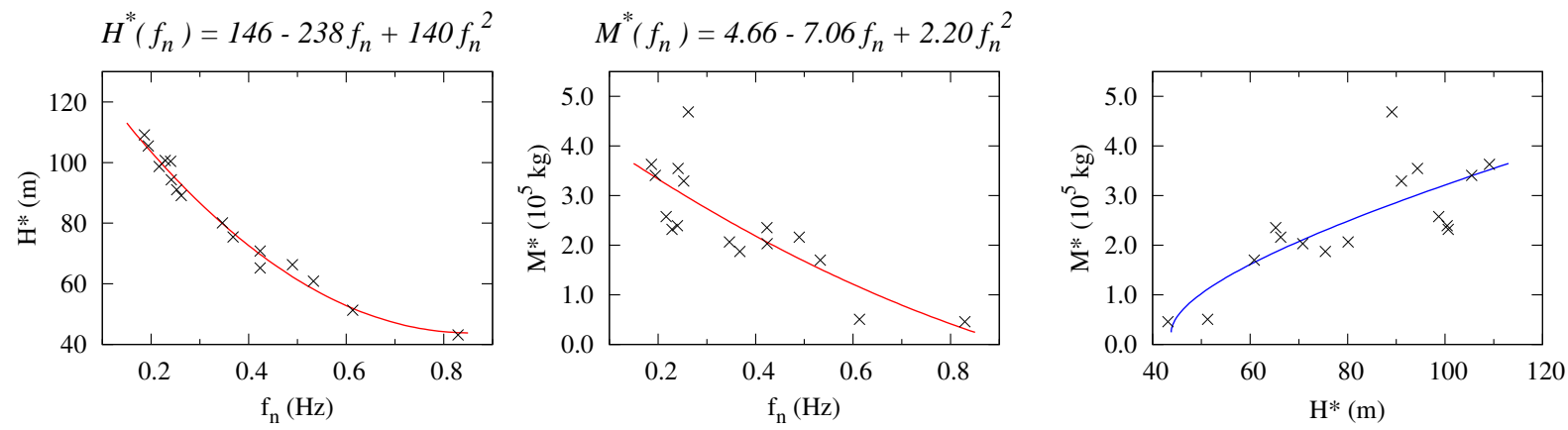

Figure 3: Modal parameters for the studied set of OWTs (crosses). Modal height and mass fitting expressions as a function of the fixed-base fundamental frequency (red lines). Relation between the modal height and mass through the fitting expressions (blue lines).

without significant errors, as shown in Fig. 3, The proposed polynomials allow to study a range of structures that follow the typical dimensions for OWT on monopile constructions instead of just a finite set of systems.

For illustration purposes, the variations in the system fundamental frequency and damping due to the SSI effects assuming a foundation consisting of a pile with $L_{p}=25 \mathrm{~m}$ and $D_{p}=4$ $\mathrm{m}$ embedded in the Nelson Field profile are plotted in Fig 4 against the fixed-base fundamental frequency. The crosses are obtained by using the real modal parameters of the studied OWT structures, while the continuous lines are obtained by assuming the relation between the modal mass or height and the fixed-base fundamental frequency given by the fitting polynomials. The results show that the use of the fitting expressions adequately follows the behaviour of the real structures.
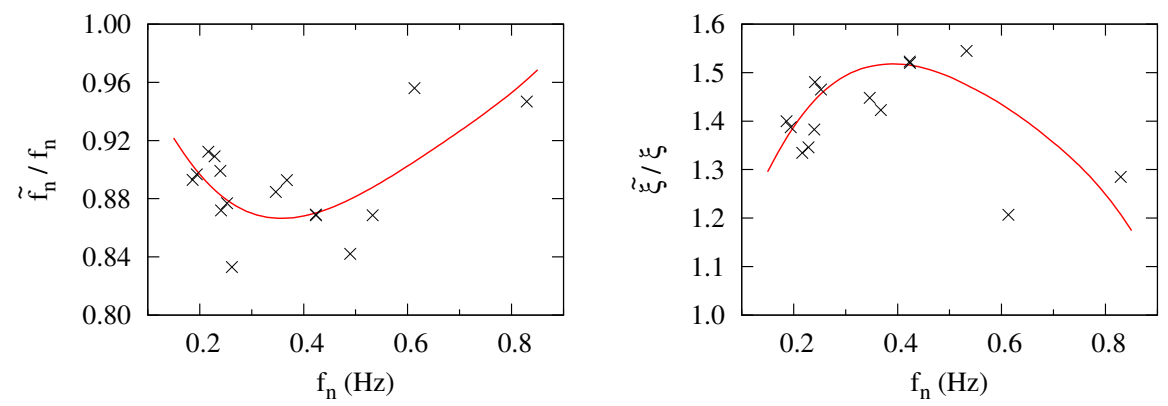

Figure 4: Variations in the system fundamental frequency and damping due to SSI effects. Comparison between the results of the fitted (line) and actual (crosses) modal parameters. Pile foundation with $D_{p}=4 \mathrm{~m}$ and $L_{p}=25$ m embedded in the Nelson Field soil profile. Structural modal damping $\xi=1 \%$.

\subsection{Influence of the modal parameters on the system dynamic properties}

In order to explain the results obtained in Fig. 4, the effects of each one of the modal parameters have to be analysed individually. Fig. 5 presents the variations in the system fundamental frequency and damping due to the SSI effects assuming a fixed value of two of the modal parameters while varying the other. Different pairs of constant parameters are employed in order to contemplate different combinations and their values are chosen in order to be within the studied ones. The same foundation dimensions and soil profile as in Fig. 4 are considered.

Attending to Fig. 5, all modal parameters increase the SSI effects if their values augment. On one hand, an increment in the system stiffness is produced if the modal mass or fixed-base 

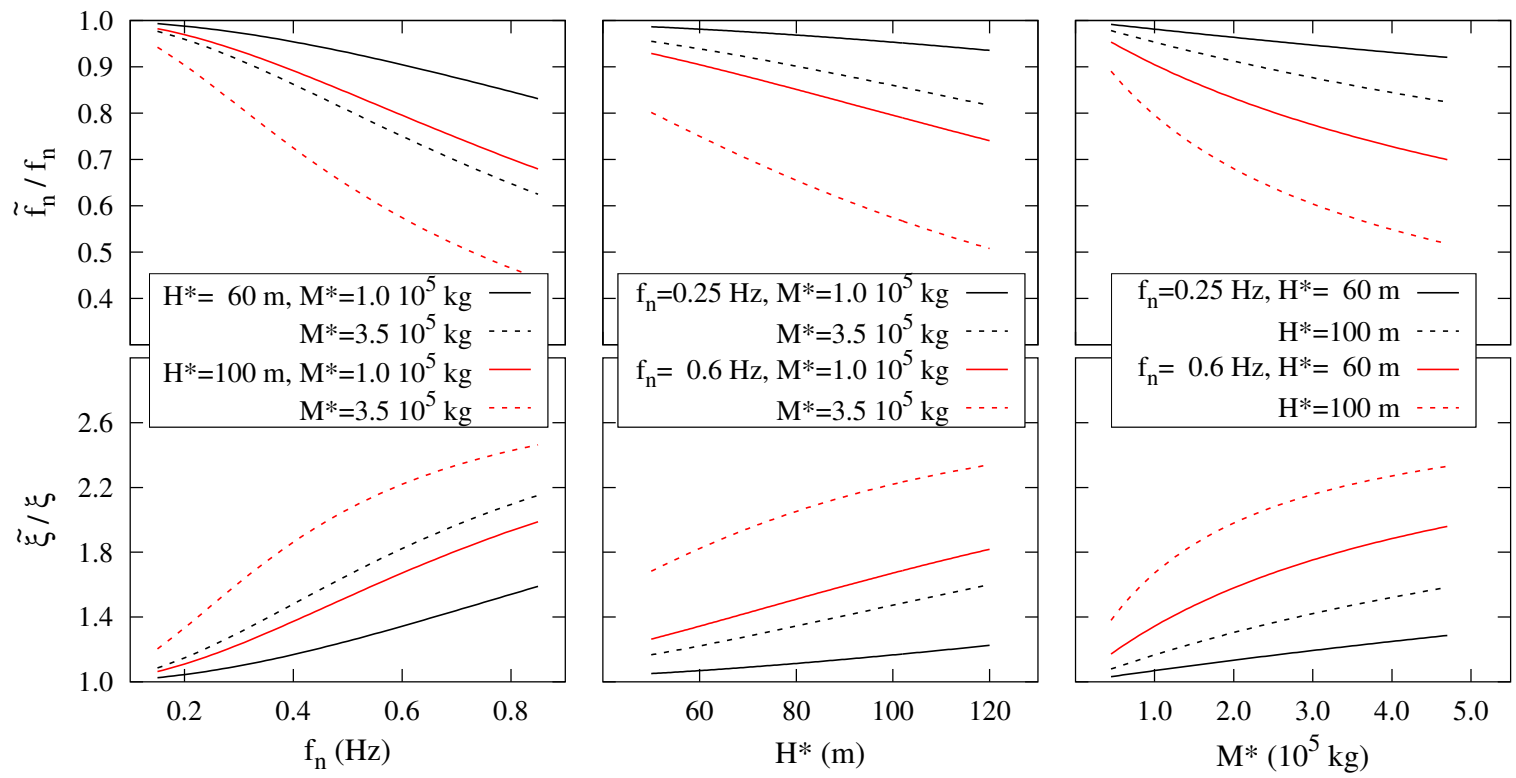

Figure 5: Influence of the modal parameters on the fundamental frequency and damping variations. Pile foundation with $D_{p}=4 \mathrm{~m}$ and $L_{p}=25 \mathrm{~m}$ embedded in the Nelson Field soil profile. Structural modal damping $\xi=1 \%$.

fundamental frequency increase while the other remains unaltered. Then, if the system stiffness augments, the soil will be relatively softer yielding higher frequency and damping variations. On the other hand, the increment of the SSI effects with the structural height has been reported in numerous studies, eg. [5, 34]. Now, the shape of the previous curves can be explained considering that in the their first part, the SSI effects increase owing to the increment in the fixed-base fundamental frequency, while in the last part the effect of the reduction in the modal mass and height has more importance.

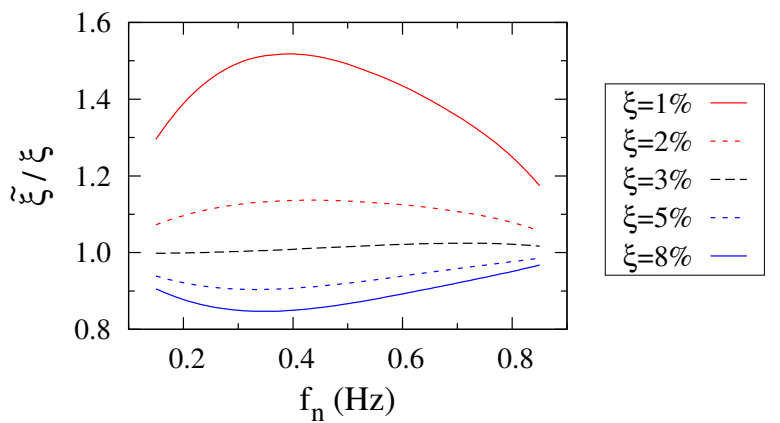

Figure 6: Influence of the fixed-base structural modal damping on the damping variations. Pile foundation with $D_{p}=4 \mathrm{~m}$ and $L_{p}=25 \mathrm{~m}$ embedded in the Nelson Field soil profile.

Finally, in Figs. 4 and 5 a structural modal damping $\xi=1 \%$ was assumed, as a typical value for studying OWT structures [36]. However, if another damping ratio is considered, the SSI effects does not change it to the same extent, as illustrated by Fig. 6. The figure shows the relation between the flexible and fixed-base damping ratios that is obtained by assuming different modal damping ratios $(\xi=1-8 \%)$. Again, the same foundation and soil as in the previous results are considered. For a modal damping $\xi=3 \%$ virtually no variations are seen. As the modal damping goes away from this value, the variations become stronger: increasing the flexible-base damping ratio for $\xi<3 \%$ and decreasing it otherwise. Nevertheless, the 
frequency range at which the system is more sensitive to the SSI effects, i.e. stronger variations, is almost the same.

\section{INFLUENCE OF THE SSI EFFECTS ON THE SYSTEM DYNAMIC PROPERTIES}

Figures 7 and 8 present the variations in the fundamental frequency and damping of the system due to the SSI effects as a function of the structure fixed-base fundamental frequency. The pile dimensions and profiles introduced in section 2 are used in order to define the foundation characteristics, while the polynomial fittings of the modal parameters presented in section 4 together with a modal damping $\xi=1 \%$ are used to define the structures.
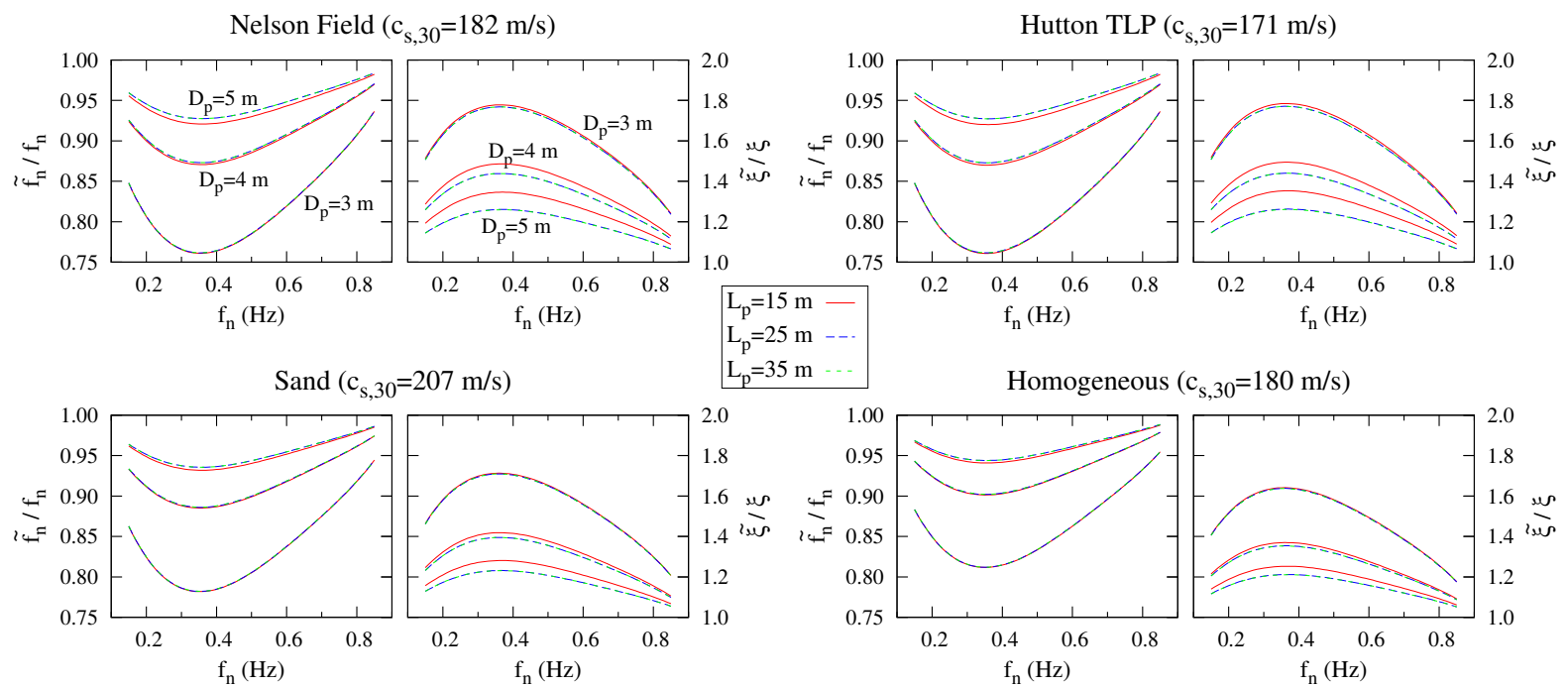

Figure 7: Variations in the system fundamental frequency and damping due to SSI effects. Results grouped by soil profile.

First, Fig. 7 shows the results corresponding to the same soil profile in each graph in order to manifest the effects of the monopile dimensions. Attending to the results, the pile diameter is found to play a significant role as its dimension is strongly related to the foundation stiffness: the higher the diameter, the stiffer the foundation yielding lower variations in the system frequency and damping. On the contrary, the pile length plays a minor role in the magnitude of the SSI effects as the results slightly vary between one value to other, being the damping variations more affected by this factor. The small influence of the pile length is explained assuming that the highest lengths are over the pile critical length.

On the other hand, Fig. 8 shows the results corresponding to all the studied soils in the same graph in order to analyse the differences between each profile. As mention before, the results marginally change depending on the pile length, thus only the curves corresponding to one length value are presented. The results for the Nelson Field, Hutton TLP and Clay profiles are virtually the same, showing that the superficial layers of the profiles are the ones that govern the studied problem. As expected, for harder soils (in terms of the $c_{s}$ of the superficial layers), the variations in the system fundamental frequency and damping are reduced. It is important to notice that significant differences are produced between the variable profiles and the homogeneous assumption, despite that all soils present close values of the $c_{s, 30}$ mean velocity. 


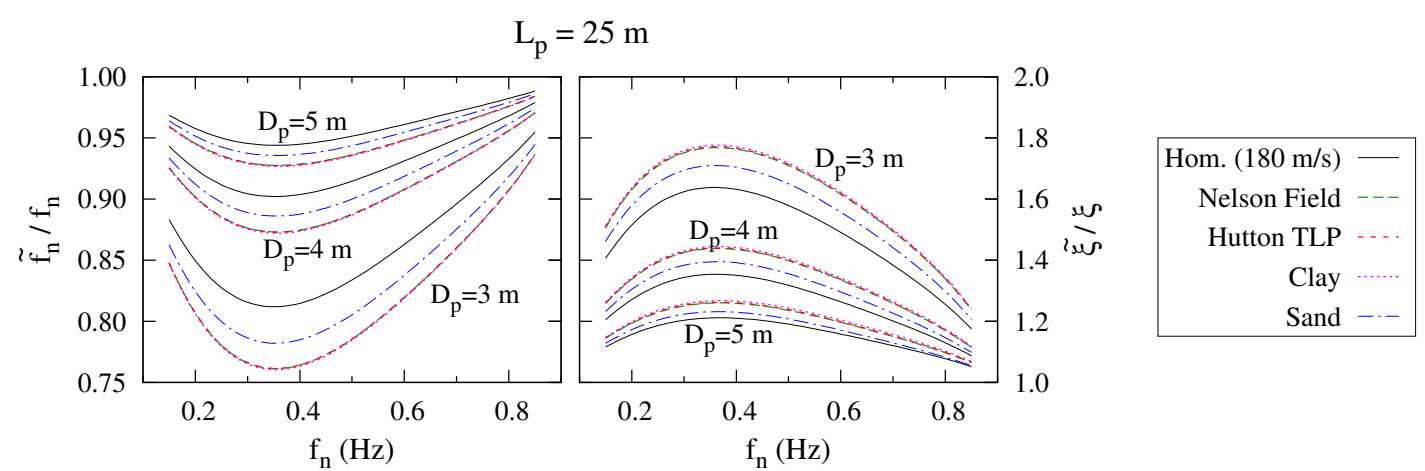

Figure 8: Variations in the system fundamental frequency and damping due to SSI effects. Results grouped by pile length.

\subsection{Influence of soil Poisson's ratio}

Fig. 9 presents the variations in the fundamental frequency and damping of the OWT system for the Nelson Field profile and considering different values of the soil Poisson's ratio. This analysis is made in order to show the influence of this parameter on the SSI effects, as high Poisson's ratios are commonly used to represent saturated soils. Values of $\nu_{s}$ between $0.35-0.49$ are used, while the rest of soil properties have the same values introduced in section 2.3 .

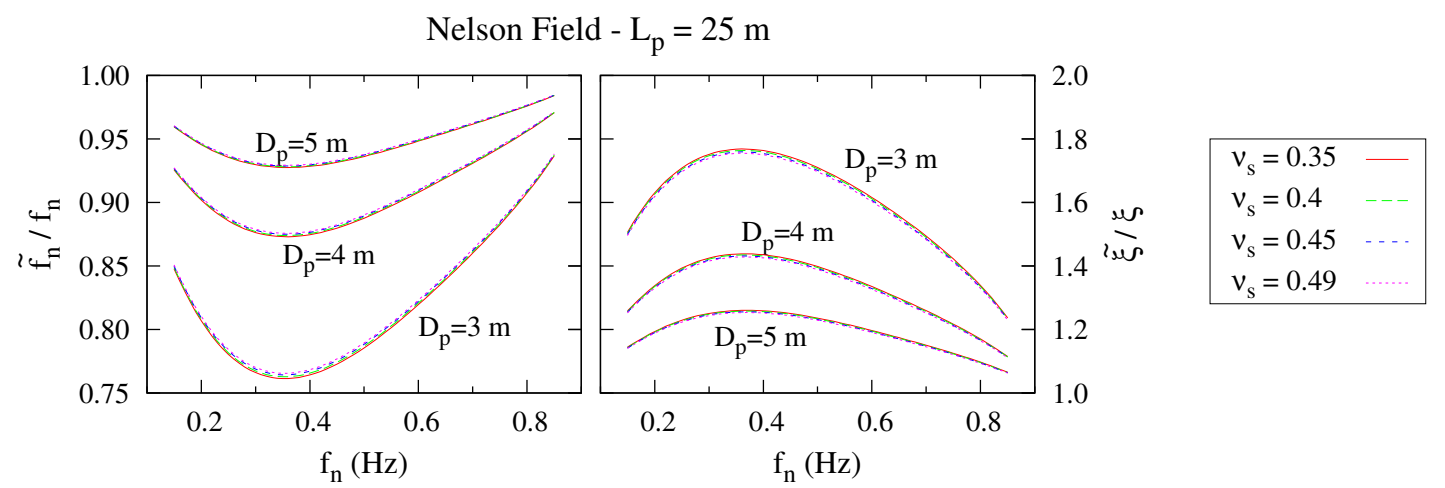

Figure 9: Variations in the system fundamental frequency and damping due to SSI effects. Influence of the soil Poisson's ratio.

The results of Fig. 9 show that increasing the soil Poisson's ratio has almost no influence on the SSI effects, as recently found by Daamgard et al. [12]. As the Poisson's ratio augments, the soil becomes slightly more rigid and, therefore, the variations in the system fundamental frequency and damping are marginally reduced. However, practically the same results are obtained for the extreme cases of $\nu_{s}=0.35$ and 0.49 . This effect is seen for all the studied profiles.

\section{CONCLUSIONS}

This work introduces a simplified substructuring model to analyse variations in the fundamental frequency and damping of OWT-monopile systems due to the SSI effects. Only the fundamental mode of the superstructure is taken into account in terms of the base shear effective modal mass and height, while the foundation stiffness modelling is addressed through impedance functions. The proposed methodology agrees well with the results of a more elaborated FEM formulation that takes all modes into account. 
The effect of real soil profiles are studied based on typical boreholes of the North Sea, while relations between the modal mass or height and the fixed-base fundamental frequency that are characteristic for the studied type of constructions are obtained based on data from real OWT systems found in the literature. The results showed reductions of 5-20\% in the system fundamental frequency, revealing the importance of the consideration of the SSI effects when analysing the dynamic behaviour of this type of structures.

Finally, the soil profile is found to play a fundamental role in the magnitude of the SSI effects, specially the superficial layers. The use of a homogeneous soil with mean properties close to the actual profile is not valid to study the SSI effects as significantly smaller variations in the system fundamental frequency and damping are produced under this assumption.

\section{ACKNOWLEDGEMENTS}

This work was supported by the Subdirección General de Proyectos de Investigación of the Ministerio de Economía y Competitividad (MINECO) of Spain and FEDER through research project BIA2014-57640-R. G.M. Álamo is a recipient of the FPU research fellowship FPU14/06115 from the Ministerio de Educación, Cultura y Deporte of Spain. The authors are grateful for this support. The authors also want to thank Esteban García, former Master student at University Institute SIANI for his data compilation.

\section{References}

[1] DNV/Risø. Guidelines for design of Wind Turbines. 2nd ed. Det Norske Veritas, Copenhagen and Wind Energy Department, Risø National Laboratory, 2002.

[2] M.B. Zaaijer. Foundation modelling to assess dynamic behaviour of offshore wind turbines. Applied Ocean Research, 28(1):45-57, 2006.

[3] S. Adhikari and S. Bhattacharya. Dynamic analysis of wind turbine towers on flexible foundations. Shock and Vibration, 19(1):37-56, 2012.

[4] L. Arany, S. Bhattacharya, S. Adhikari, S.J. Hogan, and J.H.G. Macdonald. An analytical model to predict the natural frequency of offshore wind turbines on three-spring flexible foundations using two different beam models. Soil Dynamics and Earthquake Engineering, 74:40-45, 2015.

[5] V. Zania. Natural vibration frequency and damping of slender structures founded on monopiles. Soil Dynamics and Earthquake Engineering, 59:8-20, 2014.

[6] S. Bisoi and S. Haldar. Dynamic analysis of offshore wind turbine in clay considering soil-monopile-tower interaction. Soil Dynamics and Earthquake Engineering, 63:19-35, 2014.

[7] D. Lombardi, S. Bhattacharya, and D. Muir Wood. Dynamic soil-structure interaction of monopile supported wind turbines in cohesive soil. Soil Dynamics and Earthquake Engineering, 49:165-180, 2013.

[8] W. Carswell, S.R. Arwade, D.J. DeGroot, and M.A. Lackner. Soil-structure reliability of offshore wind turbine monopile foundations. Wind Energy, 18(3):483-498, 2015. 
[9] S. Bhattacharya and S. Adhikari. Experimental validation of soil-structure interaction of offshore wind turbines. Soil Dynamics and Earthquake Engineering, 31(5-6):805-816, 2011.

[10] M. Damgaard, L.V. Andersen, and L.B. Ibsen. Computationally efficient modelling of dynamic soil-structure interaction of offshore wind turbines on gravity footings. Renewable Energy, 68:289-303, 2014.

[11] M. Damgaard, V. Zania, L.V Andersen, and L.B. Ibsen. Effects of soil-structure interaction on real time dynamic response of offshore wind turbines on monopiles. Engineering Structures, 75:388-401, 2014.

[12] M. Damgaard, L.V. Andersen, and L.B. Ibsen. Dynamic response sensitivity of an offshore wind turbine for varying subsoil conditions. Ocean Engineering, 101:227-234, 2015.

[13] M. Damgaard, L.B. Ibsen, L.V. Andersen, and J.K.F. Andersen. Cross-wind modal properties of offshore wind turbines identified by full scale testing. Journal of Wind Engineering and Industrial Aerodynamics, 116:94-108, 2013.

[14] S. Bhattacharya, N. Nikitas, J. Garnsey, N.A. Alexander, J. Cox, D. Lombardi, D. Muir Wood, and D.F.T. Nash. Observed dynamic soil-structure interaction in scale testing of offshore wind turbine foundations. Soil Dynamics and Earthquake Engineering, 54:4760, 2013.

[15] M. Damgaard, M. Bayat, L.V. Andersen, and L.B. Ibsen. Assessment of the dynamic behaviour of saturated soil subjected to cyclic loading from offshore monopile wind turbine foundations. Computers and Geotechnics, 61:116-126, 2014.

[16] H. Yu, X. Zeng, B. Li, and J. Lian. Centrifuge modeling of offshore wind foundations under earthquake loading. Soil Dynamics and Earthquake Engineering, 77:402-415, 2015.

[17] S. Bisoi and S. Haldar. Design of monopile supported offshore wind turbine in clay considering dynamic soil-structure-interaction. Soil Dynamics and Earthquake Engineering, 73:103-117, 2015.

[18] A.T. Myers, S.R. Arwade, V. Valamanesh, S. Hallowell, and W. Carswell. Strength, stiffness, resonance and the design of offshore wind turbine monopiles. Engineering Structures, 100:332-341, 2015.

[19] R.A. Kjørlaug and A.M. Kaynia. Vertical earthquake response of megawatt-sized wind turbine with soil-structure interaction effects. Earthquake Engineering \& Structural Dynamics, 44(13):2341-2358, 2015.

[20] C. Tibaldi, T. Kim, T.J. Larsen, F. Rasmussen, R. de Rocca Serra, and F. Sanz. An investigation on wind turbine resonant vibrations. Wind Energy, 2015.

[21] W. Hu, S. Thöns, S. Said, and W. Rücker. Resonance phenomenon in a wind turbine system under operational conditions. In Proceedings of the 9th International Conference on Structural Dynamics, EURODYN 2014, Porto, pages 3619-3626, 2014. 
[22] J. van der Tempel and D.P. Molenaar. Wind turbine structural dynamics - a review of the principles for modern power generation, onshore and offshore. Wind Engineering, 26(4): 211-222, 2002.

[23] M. Novak and T. Nogami. Soil-pile interaction in horizontal vibration. Earthquake Engineering \& Structural Dynamics, 5(3):263-281, 1977.

[24] J.P. Wolf. Foundation vibration analysis using simple physical models. Prentice-Hall, Englewood Cliffs NJ, 1994.

[25] J. Jonkman, S. Butterfield, W. Musial, and G. Scott. Definition of a 5-MW reference wind turbine for offshore system development. Technical Report NREL/TP-500-38060. National Renewable Energy Laboratory, 2009.

[26] D. Lombardi. Dynamics of Offshore Wind Turbines. MSc Thesis, University of Bristol, 2010 .

[27] Recommended Practice for Planning, Designing, and Constructing Fixed Offshore Platforms - Working Stress Design, Ver. 21. American Petroleum Institute, USA, 2000.

[28] Health and Safety Executive, London (UK), A J Bond, D W Hight, and R J Jardine. Design of Piles in Sand in the UK Sector of the North Sea-Report OTH 94 457. 1997.

[29] Y. Ohta and N. Goto. Empirical shear wave velocity equations in terms of characteristic soil indexes. Earthquake Engineering \& Structural Dynamics, 6(2):167-187, 1978.

[30] Eurocode 8: Design of structures for earthquake resistance. Part 5: Foundations, Retaining Structures and Geotechnical Aspects. European Committee for Standardization, Brussels, 2004.

[31] A.K. Chopra. Dynamic of structures. Theory and applications to earthquake engineering. NJ: Prentice-Hall, 2001.

[32] G.M. Álamo, A.E. Martínez-Castro, L.A. Padrón, J.J. Aznárez, R. Gallego, and O. Maeso. Efficient numerical model for the computation of impedance functions of inclined pile groups in layered soils. Submitted to Engineering Structures.

[33] R.Y.S. Pak and B.B. Guzina. Three-dimensional Green's functions for a multilayered half-space in displacement potentials. Journal of Engineering Mechanics, 128(4):449461, 2002.

[34] A.S. Veletsos and J.W. Meek. Dynamic behaviour of building-foundation systems. Earthquake Engineering \& Structural Dynamics, 3(2):121-138, 1974.

[35] C. Medina, J.J. Aznárez, L.A. Padrón, and O. Maeso. Effects of soil-structure interaction on the dynamic properties and seismic response of piled structures. Soil Dynamics and Earthquake Engineering, 53:160-175, 2013.

[36] Guideline for the Certification of Offshore Wind Turbines. Germanischer Lloyd WindEnergie GmbH, Germany, 2005. 